\title{
Application of Medical Imaging Technology in Oral and Maxillofacial Surgery
}

\author{
Takashi Ohya*1, Ichiro Sakuma ${ }^{2}$ and Kenji Mitsudo ${ }^{1}$ \\ ${ }^{1}$ Department of Oral and Maxillofacial Surgery, Yokohama City University Graduate School of Medicine, Japan \\ ${ }^{2}$ School of Engineering, The University of Tokyo, Japan
}

Received: 制: September 16, 2018; Published: 泟 September 24, 2018

*Corresponding author: Takashi Ohya, Department of Oral and Maxillofacial Surgery, Yokohama City University Graduate School of Medicine, 3-9 Fukuura, Kanazawa-ku, Yokohama, 236-0004, Japan

\begin{abstract}
Patients undergoing oral and maxillofacial surgery require recovery from dysfunction related to occlusion, eating/swallowing, speech etc. It is also essential to restore from esthetic disorders by the surgery and esthetics must not be impaired as possible even after surgery. The oral and maxillofacial surgery procedure must consider both aspects of functionality and esthetics, and the surgery must be performed with high precision. Therefore, the trend of using medical imaging technology, which has remarkably progressed in oral and maxillofacial surgery, has accelerated in recent years. Medical imaging technology enables to accurately memorize, measure, and visualize the necessary information for the operator and is indispensable for planning a surgery, assisting surgical techniques, and evaluating after surgery. This study reviews medical imaging technologies that are popular in clinical use for each major surgery in oral and maxillofacial surgery.
\end{abstract}

Keywords: Medical Imaging; Orthognathic Surgery; Dental Implant Surgery; Plate Fixation; Catheterization

\section{Introduction}

\section{Orthognathic Surgery for Jaw Deformities}

Surgeries such as Le Fort I maxillary osteotomy, sagittal split ramus osteotomy, and intraoral vertical ramus osteotomy are performed to correct jaw deformities. Occlusal position is a criterion that determines the movement extent of the jaw. Therefore, a CAD/ CAM surgical splint is prepared in advance by surgical simulation using computed tomography (CT) [1]. A CAD/CAM surgical splint is a bite plate with indentations to the teeth cusp of the upper and lower jaw. The jaw is set at the ideal occlusal position using the splint after orthognathic surgery. Moreover, previous reports have analyzed changes in facial features [2] and morphological changes in the temporomandibular joint [3] before and after the surgery, and reports on fluid dynamics have analyzes changes in airway morphology [4] after the surgery.

\section{Dental Implant Surgery for Missing Teeth}

Dental implant surgery is a procedure in which a titanium artificial tooth root is implanted in the maxilla or mandible. The ideal implant position is simulated on CT images using implant planning software, and a mouthpiece-type surgical stent is used to correspond with it. Based on these data, the surgical stent is manufactured by a milling machine or a 3D printer [5]. The surgical stent defines the position and direction of the drill to accurately reproduce the position and orientation of the simulated hole for implantation; however, this method requires sufficient maximum mouth opening. Often, the drill cannot be inserted into the hole of the surgical stent due to long drills and thick surgical stents. Hence, the overlay system [6], which automatically registers the camera and CT images, is considered a solution because the surgical stent is no longer needed. Recently, intraoral scanners have become widespread [7], and surgical stents can be designed using computer software. That is, quickly transferring the intraoral data to the laboratory has become possible and delivering the molded model has become unnecessary.

\section{Plate Fixation of Maxillofacial Fractures}

Plate fixation of maxillofacial fractures is a surgical procedure in which a deviated bone is restored to its original position against a fracture, such as in the maxilla or mandible, and the bone plate is fixed across the fracture line. It is possible to concretely assume the length, position, and direction of the bone plate by creating a real model using a 3D printer based on preoperative CT imaging data [8]. Pre-bending is also effective because it reduces the time to bend the bone plate during surgery [9]. Surgery for mandible fracture involves both intraoral and extraoral approaches. The incision approach in the oral cavity is less of an esthetic obstacle but is difficult to fix the plate and drill the screw in a narrow space. Surgery to fix the bone plate while observing the endoscopic image [10] requires operator skill, especially in cases of mandibular cervical fracture. 


\section{Catheterization for Retrograde Superselective Intra- arterial Chemoradiotherapy for Oral Cancer}

The standard treatment for oral cancer is surgery. However, patients with advanced oral cancer often refuse surgery due to postoperative cosmetic disorders and dysfunction. Retrograde superselective intra-arterial chemoradiotherapy almost completely preserves the shape of the oral cavity and face and cures the cancer [11-12]. This treatment involves making a small incision to the skin in front of and behind the ear, dissecting the artery, and inserting the catheter. A high concentration of the anticancer agent reaches the cancer site when the catheter tip is directly placed in the feeding artery of the cancer. However, when the catheter tip is not directly placed in the feeding artery, fluid dynamics analysis reveals an unstable flow of the anticancer drug [13]. Future research is needed to reduce intraoperative X-ray exposure using C-arm and contrast medium [14].

\section{Conclusion}

In the present review, we focused on orthognathic surgery, dental implantation, plate fixation, and catheterization in oral and maxillofacial surgery. Medical imaging technology not only provides surgical assistance both preoperatively and intraoperatively but also plays a major role in postoperative evaluation. Therefore, we believe that continued use of medical imaging technology will rapidly increase the quality of oral and maxillofacial surgery in the near future.

\section{Acknowledgment}

Grant-in-Aid for Encouragement of Young Scientists (B) (JSPS KAKENHI Grant Number 16K16411), JSPS, Japan.

\section{References}

1. Steinhuber T, Brunold S, Gärtner C, Offermanns V, Ulmer $H$, et al. (2018) Is virtual surgical planning in orthognathic surgery faster than conventional planning? A time and workflow analysis of an office-based workflow for single- and double-jaw surgery. J Oral Maxillofac Surg 76(2): 397-407.

2. Suh HY, Lee SJ, Lee YS, Donatelli RE, Wheeler TT, et al. (2012) A more accurate method of predicting soft tissue changes after mandibular setback surgery. J Oral Maxillofac Surg 70(10): e553-562.

\section{ISSN: 2574-1241}

DOI: 10.26717/BJSTR.2018.09.001777

Takashi Ohya. Biomed J Sci \& Tech Res

cC (P) This work is licensed under Creative Commons Attribution 4.0 License

Submission Link: https://biomedres.us/submit-manuscript.php
3. Gomes LR, Soares Cevidanes LH, Gomes MR, Carlos De Oliveira Ruellas A, Obelenis Ryan DP, et al. (2018) Three-dimensional quantitative assessment of surgical stability and condylar displacement changes after counterclockwise maxillomandibular advancement surgery: Effect of simultaneous articular disc repositioning. Am J Orthod Dentofacial Orthop 154(2): 221-233.

4. Yajima Y, Oshima M, Iwai T, Kitajima H, Omura S, et al. (2017) Computational fluid dynamics study of the pharyngeal airway space before and after mandibular setback surgery in patients with mandibular prognathism. Int J Oral Maxillofac Surg 46(7): 839-844.

5. Flügge TV, Nelson K, Schmelzeisen R, Metzger MC (2013) Threedimensional plotting and printing of an implant drilling guide: simplifying guided implant surgery. J Oral Maxillofac Surg 71(8): 13401346.

6. Wang J, Suenaga H, Yang L, Kobayashi E, Sakuma I (2017) Video seethrough augmented reality for oral and maxillofacial surgery. Int J Med Robot 13(2).

7. Fasbinder DJ (2006) Clinical performance of chairside CAD/CAM restorations. J Am Dent Assoc 137: 22S-31S.

8. Ho CT, Lin HH, Liou EJ, Lo LJ (2017) Three-dimensional surgical simulation improves the planning for correction of facial prognathism and asymmetry: A qualitative and quantitative study. Sci Rep 10(7): 40423.

9. Bai S, Shang H, Liu Y, Zhao J, Zhao Y (2012) Computer-aided design and computer-aided anufacturing locating guides accompanied with prebent titanium plates in orthognathic surgery. J Oral Maxillofac Surg 70(10): 2419-2426.

10. Anehosur V, Joshi A, Rajendiran S (2018) Endoscopic-assisted intraoral open reduction internal fixation of mandibular subcondylar fractures: initial experiences from a Tertiary-Care Maxillofacial Center in India. Craniomaxillofac Trauma Reconstr 11(3): 183-191.

11. Homma A, Onimaru R, Matsuura K, Robbins KT, Fujii M (2016) Intraarterial chemoradiotherapy for head and neck cancer. Jpn J Clin Oncol 46(1): 4-12.

12. Mitsudo K, Hayashi Y, Minamiyama S, Ohashi N, Iida M, et al. (2018) Chemoradiotherapy using retrograde superselective intra-arterial infusion for tongue cancer: Analysis of therapeutic results in 118 cases. Oral Oncol 79: 71-77.

13. Kitajima H, Oshima M, Iwai T, Ohhara Y, Yajima Y, et al. (2017) Computational fluid dynamics study of intra-arterial chemotherapy for oral cancer. Biomed Eng Online 16(1): 57.

14. Wang JC, Ohya T, Liao HE, Sakuma I, Wang TM, et al. (2011) Intravascular catheter navigation using path planning and virtual visual feedback for oral cancer treatment. Int J Med Robot 7(2): 214-224.

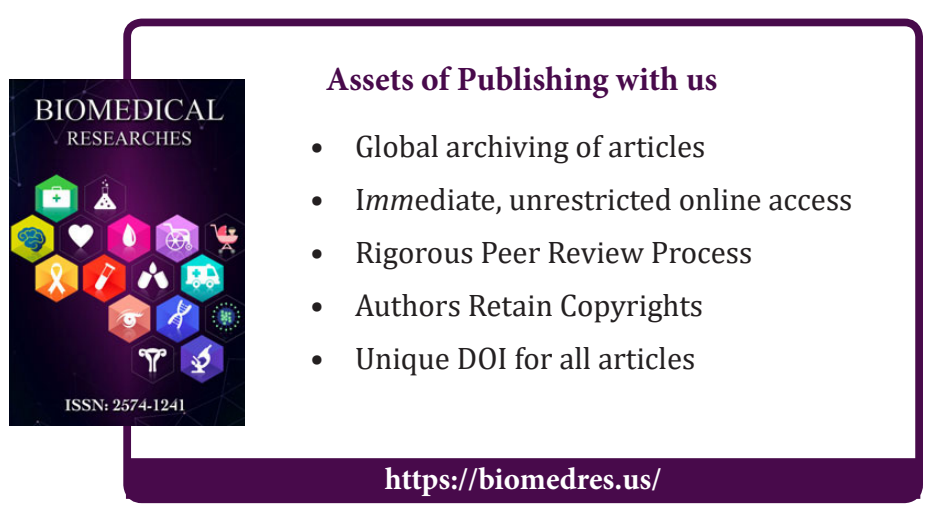

